\title{
Die komplexen Nullstellen der Besselschen Funktionen.
}

\author{
Von
}

Emil Hilb in Wüzburg.

Hurwitz ${ }^{1}$ ) hat bewiesen, daß die gerade transzendente Funktion $z^{r} J_{-\nu}(z)$ genau $2[\nu]$ konjugiert komplexe Nullstellen besitzt, wenn $[\nu]$ als größte ganze Zahl in dem stets als positiv, vorübergehend als nicht ganzzahlig angenommenen $v$ enthalten ist. Je nachdem $[v]$ gerade oder ungerade ist, gibt es unter diesen komplexen Nullstellen keine oder zwei rein imaginäre. Dagegen liegen, soweit mir bekannt ist, über die komplexen Nullstellen der Funktionen $a J_{v}(z)+b J_{-v}(z)$, wo $a$ und $b$ reelle Zahlen sind, speziell auch über die komplexen Nullstellen von $Y_{v}(z)$ so gut wie keine Resultate vor. Will man hier zu Sätzen über endliche Anzahlen kommen, so muß man sich auf die Halbebene mit positivem reellen Teil in der $z$-Ebene beschränken, für welche also, wenn

$$
\begin{gathered}
z=r e^{i \theta}, \\
-\frac{\pi}{2} \leqq \Theta \leqq \frac{\pi}{2}
\end{gathered}
$$

ist. Die so bestimmte Halbebene nennen wir im folgenden 1 und wir wählen àls Hauptwerte der vorkommenden Funktionen diejenigen $Z$ weige, die für reelle $z$ in I reell sind.

Im folgenden sollen nun die in der Theorie der Besselschen Funktionen auftretenden Fragen nach endlich vielen komplexen Nullstellen mit einfachsten Stetigkeitsbetrachtungen gelöst werden. Eine andere Ableitung würde sich aus der Methode ergeben, mit der Falckenberg ${ }^{2}$ ) und ich die Anzahl der Nullstellen der Hauptzweige der Hankélschen Funktionen bestimmten.

1) A. Hurwitz, Uber die Nullstellen der Besselsphen Funktionen, Math. Ann. 33 (1889), S. 246-266.

2) H. Falckenberg und E. Hilb, Die Anzahl der Nullstellen der Hankelschen Funktionen, Gött. Nachr., Math. phys. Kl. (1916), S. 190-196. 


\section{$\S 1$.}

\section{Hilissätze.}

Hilfssatz 1. Zwischen zwei die Null nisht einschließenden reellen oder rein imaginären Nullstellen $z_{1}, z_{2}$ einer im Intervalle $\left\langle z_{1}, z_{2}\right\rangle$ reellen Lösung $y_{1}$ der Differentialgleichung

$$
\frac{d}{d z} z \frac{d y}{d z}+\left(z-\frac{y^{2}}{z}\right) y=0
$$

der Besselschen Funktionen hat jede andere in $\left\langle z_{1}, z_{2}\right\rangle$ reelle Lösung $y_{9}$ von (3) genau eine Nullstelle.

Der Beweis folgt unmittelbar aus

$$
z\left[y_{1} \frac{d y_{z}}{d z}-y_{2} \frac{d y_{2}}{d z}\right]=C
$$

wo $C$ eine reelle Konstante ist.

Da ferneI

$$
J_{v}(z)=\left(\frac{z}{2}\right)^{n} \sum_{n=0}^{\infty} \frac{(-1)^{n}\left(\frac{z}{2}\right)^{2 n}}{n ! \Gamma(v+n+1)}
$$

keine rein imaginären Nullstellen haben kann, weil in $z^{-v} J_{v}(z)$ für rein imaginäres $z$ alle Summanden der rechten Seite von (5) positiv sind and $\mathrm{da} e^{ \pm \frac{y \pi i}{2}} J_{y}(z)$ und $e^{\mp_{2}^{r, i}} J_{-, v}(z)$ für rein imaginäre $z$ reell sind, so folgt aus Hilfssatz 1

Hilfssatz 2. $J_{-i}(z)$ kann höchstens zwei rein imaginäre Nullstellen haben, die übrigens tonjugiert sind.

In entsprechender Weise folgt

Hilfssatz 3. Zwischen 0 und $\frac{\pi}{2}$ und zwischen 0 und $\frac{-\pi}{2}$ kann $J_{-,}(z)$ höchstens je eine reelle Nullstelle haben.

Die kleinste positive Nullstelle der geraden Funktion $z^{-v} J_{v}(z)$ ist nämlich größer als $\frac{\pi}{2}$, da in

$$
J_{\nu}(z)=\frac{1}{\sqrt{v}} \frac{\left(\frac{z}{2}\right)^{v}}{\Gamma\left(v+\frac{1}{2}\right)} \int_{-1}^{+1}\left(1-u^{2}\right)^{v-\frac{1}{2}} \cos z u d u
$$

der Integrand für reelle positive $z<\frac{\pi}{2}$ positiv ist.

Wir wollen nun einige Sätze über das asymptotische Verhalten der Nullstellen ableiten und gehen dazu von den in der Halbebene I für große $|z|$ gültigen angenäherten Darstellungen 
E. Hib.

$$
J_{v}(z)=\sqrt{\frac{2}{x z}} \cos \left(z-\frac{2 y+1}{4} \pi\right), \quad I_{-y}(z)=\sqrt{\frac{2}{\pi z}} \cos \left(z+\frac{2 y-1}{4} \pi\right)
$$

aus. Die Wurzeln der Gleichung

$$
a J_{v}(z)+b J_{-m}(z)=0
$$

in der Halbebene I liegen, sofern ihr absoluter Betrag groß genug ist, in beliebiger Nähe der entsprechenden Warzeln der Gleichung

$$
a \cos \left(z-\frac{2 y+1}{4} \pi\right)+b \cos \left(z+\frac{2 y-1}{4} z\right)=0
$$

Sind also $a$ und $b$ reell, so läBt sich eine Zahl $R$ angeben, daB für alle positiven $\%$, die unterhalb einer festen Zahl $N$ liegen, alle Wurzeln von (8) in 1 , deren absolnter Betrag $R$ übersteigt, reell sind, da aus einer einfachen Wurzel von (9) bei genügend großem absoluten Betrage nicht zwei konjugiert komplexe, in I gelegene Wurzeln von (8) hervorgehen können. Man kann dieses auch daraus erschließen, daB bei reellen $a$ und $b$ nach Hilfssatz 1 eine reelle Wurzel von (8) zwischen zwei reellen positiven Wurzeln von $J_{*}(z)$ liegen muB, woduroh die aus $(9)$ bei genügend groBem absoluten Betrage angenähert erhaltenen Nullstellen von (8) alle eingeordnet sind, Es gilt also

Hilfssatz 4. Fut alle positiven $y$ unterhalb einer festen Zahl $N$ gibt es eine Zahl $R$, daß (8) bei reellen a und $b$ in der Halbebene I keine komplexen Wurzeln vom absoluten Betrag grofer als $R$ hat.

Da $z^{\eta} J_{-\eta}(z)$ eine ganze gerade Funktion ist, so folgt

Hilfssatz 5. Die komplexen Nullstellen von $J_{-v}(z)$ liegen für $v<N$ dem absoluten Betrage nach unterhalb eine. festen Zahl $R$.

Wir beweisen schlieblich

Hilfssatz b. Ist y keine ganze Zahl, sind a und b reell, aber $a \neq 0$, so hat (8) keine rein imaginäre Wurzel $\xi i$.

Wäre nämlich

$$
a(\xi i)^{2} \cdot \frac{J_{n}(\xi i)}{(\xi i)^{2}}+b(\xi i)^{v} J_{m v}(\xi i)=0
$$

wobei nach $(5) \frac{J_{y}(\xi i)}{(\xi i)^{p}}$ und $(\xi i)^{\nu} J_{-x}(\xi i)$ reell sind, so wäre

$$
a \sin v \pi \frac{J_{r}(\xi i)}{(\xi i)^{r}}=0
$$

als imaginärer Teil der linken Seite von (10), was aber unter den gemachten Voraussatzungen unmöglich ist, da $\mathscr{J}_{v}(z)$ bei positivem $v$ keine imaginären Nullstellen hat. 
$\S 2$.

\section{Beweis des Satzes von Hurwitz.}

$z^{y} J_{-y}(z)$ ist eine ganze transzendente Funktion von $z$, die, wenn $v$ gleich einer ganzen Zahl $k$ ist, in $(-1)^{k} z^{k} J_{k}(z)$ übergeht, also nach (5) in $z=0$ eine $2 k$-fache Nullstelle hat. Alle Nullstellen von $J_{k}(z)$ sind reell ${ }^{y}$ ), Lassen wir nun $y$ von $k$ aus stetig wachsen, so $\operatorname{kann} z^{v} \cdot J_{-v}(z)$ komplexe Wurzeln entweder durch Zusammenfallen reellex Wurzeln oder aus dem Unendlichen erhalten.

Das letztere ist wegen Hilfssatz (5) unmöglich. Da $J_{-, v}(z)$ der Differentialgleichung (3) genügt, so kann es nur in der singulären Stelle $z=0$ eine mehrfache Wurzel haben, $z^{v} J_{-v}(z)$ hat aber, wenn $v$ keine ganze Zahl

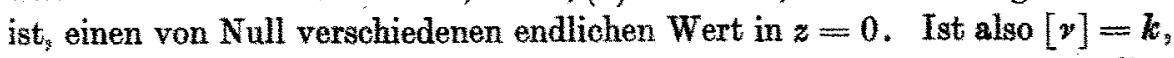
so können höchstens die für $v=k$ in Null zusammengefallenen $2 k$ Nullstellen komplex gewarden sein und es müssen auch mindestens $2 k-2$ komplex sein, da nacb Hilfssatz 3 von den $2 k$ in $z=0$ vereinigten Nullstellen, wenn $v$ sich von $k$ aus stetig ändert, höchstens eine positiv und eine negativ reell werden kann. Aber auch dieses letztere ist unmöglich, da für $y=k+1$ ja $2 k+2$ Nullstellen von $z^{v} J_{-y}(z)$ nach Null fallen, von denen wegen Hilfssatz 3 nur zwei vorher reell sein konnten. Ist also $y$ keine ganze Zahl, $[v]=k$, so hat $z^{y} J_{-y}(z) 2 k$ komplexe Wurzeln, die, weil $z^{y} J_{-\gamma}(z)$ reelle Koeffizienten hat und gerade ist, zu je vieren die Ecken eines Quadrates mit zu den Achsen parallelen Seiten bilden, es sei denn, daß Nullstellen auf der rein imaginären Achse liegen. Nach Hilfssatz 2 kann es höchstens zwei solche geben, ist also [v] ungerade, also $2[\nu]$ nicht durch 4 teilbar, so muß es zwei rein imaginäre Nullstellen geben, während wenn $[v]$ gerade ist, es keine rein imaginäre geben kann, da $2[v]-2$ nicht durch 4 teilbar ist.

\section{$\$ 3$.}

\section{Komplexe Nullstellen von $a J_{v}(z)+b J_{-v}(z)$.}

Wir nehmen $a$ und $b$ als reell, $b \neq 0$ an und beschränken $z$ auf die Halbebene 1 mit positivem Realteil. Von

$$
y=a J_{v}(z)+b J_{-\rightarrow}(z)
$$

betrachten wir nur den Hauptwert in $I$ und nehmen an, um nicht auf Bekanntes zurückzukommen, daB $v$ eine nicht ganze, aber positive Zahl sei, die wir jetzt als fest annehmen. Wir setzen zunächst $a=0$, daun hat $y$ nach $\$ 2$, wenn $[\nu]$ eine gerade Zahl ist, $[v]$ komplexe Nullstellen

3. N. Nielsen, Handbuch der Zylinderfunktionen, S. 161. 
mit positiven Realteil, wenn $[v]$ ungerade ist, $[v]-1$ solche Nullstellen, dazu noch zwei rein imaginäre. - Verändern wir $a$, so kann, sobald $a \neq 0$,

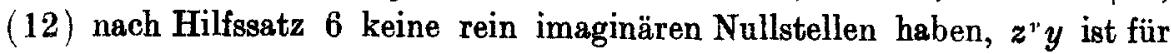
$z=0$ endlich und von Null verschieden. Wie im $\$ 2$ kann sich also bei Abänderung von $a$ die Anzahl der komplexen Nullstellen nicht ändern, und wir haben nur noch zu sehen, was bei ungeradem [ $\nu$ ] aus den beiden rein imaginären Nullstellen $\pm \xi i$ von $J_{-v}(z)$ wird, wobei es genügt, $|\varepsilon|$ beliebig klein zu wählen, wenn $\frac{a}{b}=\varepsilon$ gesetzt wird. Wir bestimmen daher die zu $\pm \xi i$ benachbarten Wurzeln $\pm \xi i+\delta$ der Gleichung

$$
\varepsilon+\frac{z^{v} J_{-v}(z)}{z^{\nu} J_{v}(z)}=0
$$

wobei also $|\delta|$ mit $|\varepsilon|$ nach Null konvergiert. Wir bezeichnen nun mit $\equiv$ „Gleichheit abgesehen von einem positivem reellen Faktor", dann ist wegen (2) für reelle positive $y$

(14) $( \pm i y)^{v} J_{v}( \pm i y) \equiv e^{+\pi i v}, \quad( \pm i y)^{\prime} J_{-v}( \pm i) \equiv-\left(-y^{2}+\xi^{2}\right)$ da bei ungeradem $[v]$ nach $(5) z^{v} J_{-v}(z)$ in Null einen negativen reellen Wert hat. Setzt man daher $\pm \xi i+\delta$ für $z$ in (13) ein, so erhält man bei Vernachlässigung der höheren Potenzen von $\delta$

$$
\varepsilon \equiv \pm \frac{2 \xi i \delta}{e^{+\pi i v}}
$$

also wenn

$$
\begin{gathered}
\delta=\delta_{1}+i \delta_{2}, \\
\delta_{1}=\varepsilon \sin v \pi \equiv-\varepsilon,
\end{gathered}
$$

da $[v]$ ungerade ist.

Bei ungeradem $[\nu]$ rücken also die beiden rein imaginären Nullstellen in die Halbebene I dann und nur dann hinein, wenn $\frac{a}{b}$ negativ ist, im anderen Falle rücken sie in die andere Halbebene. Man hat also

Satz. Sind a und $b$ reell, ist ferner $b \neq 0$, so besitzen die Hauptwerte der Funktionen $a J_{\nu}(z)+b J_{-v}(z)$ bei geradem $[v]$ genau $[v] k 0 m$ plexe Nullstellen mit positivem Realteil, bei ungeradem [v] entweder $[v]-1$ oder $[v]+1$, je nachdem $\frac{a}{b}$ positiv oder negativ ist.

\section{$\S 4$.}

Anzahl der komplexen Nullstellen von $Y_{y}(z)$ in der Halbebene $I$.

Es ist

$$
Y_{\nu}(z)=\frac{1}{\sin v \pi}\left(\cos v \pi J_{v}(z)-J_{-v}(z)\right)
$$


Ist $y$ zunächst nicht ganzzahlig, so hat nach $\$ 3$ bei geradem [ $\nu]$ der Hauptwert von $Y_{b}(z)[v]$ komplexe Nullstellen in der Halbebene $\mathrm{I}$, bei ungersdem $[v]$ aber $[v]-1$ oder $[v]+1$ solche Nullstellen, je nachdem $y-[y]<\frac{1}{2}$, also ons $y \pi<0$ oder $y-[y]>\frac{1}{2}$, also $\cos v \pi>0$ ist. Es haben daher auch, wenn $v$ eine ganze Zahl $2 n$ oder $2 n+1$ wird, $F_{2 n}(z)$ und $Y_{2 n+1}(z)$ in der Halbebene I $2 n$ komplexe Nullstellen, da für irgendeine ganze Zahl $k$

$$
\begin{aligned}
z^{k} n Y_{k}(z) & =2 z^{k} J_{k}(z) \ln \frac{z}{2}-\sum_{s=0}^{\infty}(-1)^{*} \frac{\left(\frac{z}{2}\right)^{2 k+2 z}}{s !(s+k) !}\left(\frac{\Gamma^{\prime}(z+1)}{\Gamma(s+1)}+\frac{\Gamma^{\prime}(s+k+1)}{\Gamma(s+k+1)}\right) \\
& -\sum_{s=0}^{-1} \frac{(n-s-1) !}{s !}\left(\frac{z}{2}\right)^{i_{s}}
\end{aligned}
$$

für $z=0$ einen endlichen von Null verschiedenen Wert hats Gibt man $z$ einen rein imaginären Wert $\xi \xi$, so wird der rein imaginäre Tenl von (19) $(\xi i)^{k} J_{k}(i \xi) \pi i$, ist also, da $J_{k}(z)$ keine rein imaginären Nullstellen hat, sicher von Null verschieden, so daB unter den $2 n$ komplexen Nullstellen von $Y_{a_{n}}(z)$ und $Y_{3 n+1}(z)$ in der Halbebene $I$ keine rein imaginären sind. Ebenso folgt, dab die Hauptwerte von $a Y_{n s}(z)+b T_{3 n}(z)$ und $a Y_{9 n+1}(z)$ $+b J_{2 n+1}(z)$ in der Halbebene I genan $2 n$ komplexe Nullstellen bei reellen $a$ und $b$ baben.

(Aingeganger am 14. April 1922, 